\title{
Dimensiones que influyen en el emprendedurismo por parte de los alumnos de la licenciatura en creación y desarrollo de empresas (LCyDE) de la UAEH
}

\author{
Dimensions that influence entrepreneurship by the pupils of the degree in business creation and \\ development (LCyDE) of the UAEH
}

Juan J. Aguilar-Lugo-Marino ${ }^{a}$

\begin{abstract}
:
The aim of this work is to know how much similarity have other countries and regions, and the motivators compared with students of the degree creation and business development using the work of Maryland (2019), where they surveyed entrepreneurs seven regions and countries with an instrument of seven dimensions; applying the instrument to the students being the correlation stronger with entrepreneurs of Singapore more than with entrepreneurs from Latin America.
\end{abstract}

Keywords:

Motivation of entrepreneurship, creation and business development

\section{Resumen:}

El objetivo del presente trabajo es conocer qué tanta similitud tienen con otros países y regiones los motivadores que tienen los alumnos de la Licenciatura en Creación y Desarrollo de Empresas (LCyDE) utilizando el trabajo de Maruland, Montoya \& Vélez (2019) donde ellos encuestaron a emprendedores de siete regiones y países con un instrumento de siete dimensiones; aplicando el instrumento a los alumnos la correlación más fuerte fue con los emprendedores de Singapur, más no con los emprendedores de América Latina..

\section{Palabras Clave:}

Motivación para emprender, creación y desarrollo empresarial

\section{Introducción}

La palabra "empresa", de origen italiano, desde siempre ha evocado la idea de emprender una acción con ciertos objetivos; en su uso hay muchas veces un dejo de admiración por alguien que emprende o inicia una empresa, en términos de intentar lograr algo con esfuerzo. Esta idea que desde las revoluciones industriales europeas acompañaría los esfuerzos del capital por diseñar procesos productivos que permitirían contar con nuevas formas de organización del trabajo, conservaría aquella cierta dosis de subjetividad admirativa, en términos de los retos que implican los esfuerzos por lograr los objetivos buscados (Reynoso, 2014).
Es importante, al tocar este tema, enfatizar que México es un gran semillero de emprendedores, de acuerdo con la Organización para la Cooperación y el Desarrollo Económico (OCDE), "México es el segundo mejor país para emprender del bloque y la economía que más empresas crea al año (Márquez, 2018)", ya que también según datos que obtuvo Uribe (2018) de la Secretaría de Economía, que es el organismo que fomenta la productividad del país, -ya que la necesidad de favorecer la creación de empresas y su consideración como motor de crecimiento económico se ha convertido en el objetivo final de una gran parte de organismos públicos (Alonso, González, \& Nieto, 2015)- cada mes se crean unas 35,000 empresas, que en un $6 \%$ fueron gestadas por jóvenes de entre 18 y 24 años de edad. Una edad que debe ser 
propicia para la creación de empresas, el joven puede haber egresado de alguna carrera o está a punto de hacerlo, por lo que tiene conocimientos de alguna profesión y en muchas ocasiones sus compromisos económicos no son altos por lo que no es tan complicado el tener ingresos variables. $Y$ algo muy importante de nuestra juventud es la innovación, que es un elemento básico para el éxito, el crear algo diferenciador que llegue a impactar el mercado donde se pueda posicionar.

Los aportes e investigaciones presentadas en el marco de la innovación empresarial dan cuenta de que las pymes han entrado en la generación de conocimientos innovadores para crear productos de calidad y asegurar su competitividad, como lo menciona Díaz, Urbano, \& Hernández (2005: 209) "se debe fomentar la capacidad competitiva, a través de la innovación, la capacidad emprendedora y la flexibilidad del sistema productivo".

En algunos sectores se establecieron estrategias de cambio o mejoras innovadoras en mayor proporción que las grandes empresas (Bonilla, 2014), esto tiene su lógica, la persona que está al frente de las pymes tiene un contacto cercano con los clientes, se puede casi asegurar que es "face to face" por lo que tiene, de primera mano, conocimiento de las tendencias del mercado, y no sólo eso, por lo reducida de su estructura organizacional puede hacer cambios innovadores con mayor facilidad y con medición de resultados en poco tiempo. Cosa que no es tan fácil en las grandes empresas, aunque cuenten con un cuerpo de creativos dentro de la organización, en ocasiones su estructura es muy vertical.

\section{DESARROLLO}

\section{El emprendurismo}

Analizando los aspectos de creación con innovación de empresas, Schumpeter, citado por Hernández A. (2010) menciona que existen cinco tipos posibles que son: la introducción a un nuevo producto, la creación de un proceso distinto de producción, el incursionar en un nuevo mercado, la obtención de una nueva fuente de insumos $u$ otros que pueden ser sustituidos por los existentes y cambios en la organización de la empresa; estos ejemplos nos ilustran lo ya planteado, los cambios son más sencillos en las pymes que en las grandes empresas.

Usualmente cuando se quiere comenzar una empresa lo primero que se hace es visualizarse como un gran emporio, viajando, comprando los mejores autos, la casa de nuestros sueños, etc., pero lo que se ignora que para llegar a esos términos se tiene que pasar por caminos "empedrados y además con baches". En la mayoría de los procesos de incubación se incluyen temas que ayudan a motivar con el típico "tú puedes", pero no preparan realmente para salir a una batalla cruda y sangrienta, como es la del mercado, donde normalmente sobrevive el más fuerte o el más astuto.
El emprendedor debe conocer el lado oscuro del emprendimiento — como lo llama Zúñiga (2013, Pág. 46) - donde pueda comprender y además experimentar todos los obstáculos que tendrá que sortear al momento de comenzar su proyecto.

Este lado oscuro va desde el entender muy bien lo que significa pagar tus impuestos en tiempo y forma, ya que resulta difícil asumir que tenemos que ceder parte de nuestras utilidades al fisco, lo cual, además, es algo de lo cual deberíamos estar orgullosos.

Otro elemento del lado oscuro puede ser la actitud que tienen los demás al saber de nuestro proyecto, donde se originan frases como: "no pierdas tu tiempo mejor busca un empleo", "esa idea ya se me había ocurrido y no funcionó", "no creo que resulte ya existen muchas empresas dedicadas a lo que tú quieres hacer". ¿Quién prepara al emprendedor para estas etapas, o es que tiene que aprenderlas sobre el camino? (Ibidem: 46).

\section{Licenciatura en Creación de Empresas de la UAEH}

La creación de empresas ha ido creciendo en importancia como instrumento para transferir a la sociedad el conocimiento generado en las universidades (Rodeiro, Fernández, Otero, \& Rodríguez, 2008). La universidad juega un papel muy importante en las actividades socioeconómicas de un país, en tanto que, además de generar actividades propias de enseñanza-aprendizaje e investigación y desarrollo, participa en la creación de nuevas empresas (Herrera, Salas, Domínguez, \& Torres, 2015, pág. 120); el fomento de la cultura emprendedora es uno de los aspectos de la vinculación con un mayor seguimiento entre las IES mexicanas (Santamaría \& Brunet, 2014) , esto no sólo como una actividad de vinculación; para tener un mejor soporte en esta actividad es importante contar con programas educativos que sean los artífices, junto con los investigadores de la transferencia de tecnología a la sociedad. Si bien es cierto, según datos de Santamaria \& Brunet (Ibid., 11) un 96\% de las universidades públicas estatales realiza actividades de fomento de la cultura emprendedora y un $87.5 \%$ de ellas incluye carreras con materias que fomentan esta cultura, lo ideal son programas educativos que íntegramente abordan la creación y desarrollo de empresas. "La Universidad, al transmitir conocimientos, proporciona a los empleadores las herramientas básicas de su éxito (Garcia, 2011)".

Cabe resaltar que este tipo de programa debe de tener un fuerte vínculo con empresas competitivas, de hecho el alumno debería "vivir" en estos organismos, ya que este aprendizaje en la empresa es muy particular, especialmente cuando consideramos la "forma propia de hacer las cosas" que surge de la cultura organizacional, agregada a los productos y servicios que ofrece la 
empresa, vinculados a las características particulares del entorno comercial o industrial que los rodea y matizados con el saber, saber hacer y el saber ser de las personas que trabajan en ella. Esta mezcla es única, como únicas son las personas que se desempeñan laboralmente en una organización, lo cual hace que este aprendizaje propio no pueda darse en una institución universitaria o de formación continua.

Visualizando esto la Universidad Autónoma del Estado de Hidalgo en su Guía para el Rediseño y Presentación de Programas de Licenciatura (UAEH, 2005), ha creado tres escenarios que son: el Áulico, Virtual y Real, para que los alumnos al combinar éstos, tengan una formación más sólida, el énfasis es en el último escenario: formarse dentro de la empresa. Esto fortalece a nuestros egresados adquiriendo una experiencia propia de su ejercicio profesional, algo tan demandado por los empleadores: la experiencia. Logrando con esto programas educativos con temas pertinentes de los cursos que se imparten (Quintero, 2003).

Es por eso que en la autónoma del estado de Hidalgo, después de realizar estudios de pertinencia y factibilidad para conocer qué carreras son las más propicias, una alternativa que surgió con gran beneplácito fue Licenciado en Creación y Desarrollo de Empresas, sobre todo visualizando el quehacer universitario, ya que es necesaria una carrera diseñada para el apoyo de las empresas existentes con alumnos vinculados con el sector productivo y con una visión emprendedora.

La Licenciatura en Creación y Desarrollo de Empresas de la $\mathrm{UAEH}$, es un Programa Educativo que forma profesionales multidisciplinarios, capaces de enfrentar los retos sociales, resolver la problemática que enfrentan las empresas independientemente de su tamaño y ser detonador del surgimiento, desarrollo y crecimiento, contribuyendo a mejorar la calidad de vida de su entorno; en vista de que los sectores secundario y terciario ocupan el porcentaje más alto, el egresado tendrá mayores posibilidades para generar una organización preparada para satisfacer las necesidades de estas ramas productivas.

Es de vital importancia, estimular una nueva cultura empresarial basada en la innovación tecnológica, la competitividad y la integración de redes de negocios, potencializando los recursos naturales con que cuenta el país, el Estado y en particular la región del Valle del Mezquital -donde se ubica-, así como aquellas áreas de producción y servicios no explotados actualmente.

Otra necesidad prioritaria, es el conocimiento en el proceso de incubación y desarrollo de proyectos emprendedores que generen fuentes de empleo, dando un impulso y crecimiento al estado de Hidalgo, a nivel nacional e internacional de acuerdo con el impacto de inserción en el campo laboral del profesionista (UAEH, 2014).

Una gran ventaja que también se ofrece en este programa educativo es que el último semestre se realiza dentro de una empresa, así se fortalece el escenario real de los futuros profesionistas.

\section{La universidad en la Triple Hélice: Universidad-Empresa-Gobierno}

Las universidades se encuentran en búsqueda permanente de comunicación con el Estado y el sector privado para el intercambio de necesidades de ofertas y requerimientos, la mayoría de las comunicaciones del sector universitario se realizan con los gobiernos locales y el sector pyme (pequeña y mediana empresa), por lo que se debe intensificar o mejorar la comunicación con empresarios (Rodríguez, Acuña, Rojas, \& Lobato, 2015). Las universidades son piezas claves para el desarrollo, porque su acción está necesariamente vinculada a otras instancias como la empresa privada grande y pequeña, el capital financiero, los organismos gremiales y demás activos nacionales, la pertinencia de la educación se garantiza a través de la oferta educativa contextualizada, con lo que la universidad adquiere su sentido pleno como un actor importante del país.

El desarrollo de la actividad productiva debe estar ligado al quehacer académico y a la actividad empresarial, y para propiciar este desarrollo se deben establecer fuertes vínculos entre estas instituciones.

La interacción universidad-empresa se ha fortalecido en muchos países en las últimas décadas, debido al incremento del apoyo gubernamental a los centros de investigación y producción de conocimientos, al decidido interés de algunas empresas privadas en establecer equipos interdisciplinarios para los procesos de investigación y desarrollo requeridos en sus áreas de negocios y al cambio de mentalidad en la gestión de las instituciones académicas por parte de sus dirigentes. Por ello hoy se vive un profundo cambio de los modelos sociales respecto a la gestión tradicional de la universidad, pasando de una visión esencialmente académica a una nueva visión empresarial complementaria.

Incluso en México, las grandes cadenas están buscando alternativas de apoyo a PYMES, como es el caso del área de compras de Walmart, que busca productos que ya demostraron que tienen valor e innovación. Cuando los encuentran, la trasnacional invita a sus creadores a formar parte del programa Adopta una Pyme, en el que reciben capacitación por un año en temas como logística, mercadotecnia, sustentabilidad, pagos, facturación y negociación, entre otros. Una vez concluido, pueden vender sus productos en Walmart, Bodega Aurrera, Superama y Sam's Club que son unas de las 3,134 unidades en el país que tiene el grupo (Gasca, 2018). 
El actuar de la universidad como una unidad de negocios en los países industrializados ha obedecido, en gran parte, a razones de fuerza mayor, por la necesidad de comunicarse con un sector económico que, cada día más, le plantea nuevos retos de desarrollo, la invita a participar en grupos de investigación conjuntos para la solución de problemas y que, además, está en alerta permanente sobre la producción académica potencialmente comerciable que pueda surgir desde el interior de los laboratorios y centros de investigación de la universidad (Rodríguez, Acuña, Rojas \& Lobato, Op. Cit.:18).

Como ocurre en México con la creación del RENIECyT (CONACyT, 2019), que es el Registro Nacional de Instituciones y Empresas Científicas y Tecnológicas, siendo un instrumento de apoyo a la investigación científica, el desarrollo tecnológico y la innovación del país a cargo del CONACYT a través del cual identifica a las instituciones, centros, organismos, empresas y personas físicas o morales de los sectores público, social y privado que llevan a cabo actividades relacionadas con la investigación y el desarrollo de la ciencia y la tecnología en México.

Aspectos que motivan para crear una empresa desde diversas latitudes

Centrando la atención en algunos trabajos desarrollados en la línea de investigación de Modelos de Proceso de Creación de Empresas, establecen como factor esencial del proceso de puesta en marcha de empresas, la oportunidad de negocio (Mejía, 2012). Donde el emprendedor, despliega sus capacidades y habilidades, para eso se conjugan varios elementos, como son: la necesidad, el entorno, la estrategia y el tiempo para resolver problemas ya existentes o creados por el mismo empresario.

Pero, resulta de gran importancia saber cuáles son los factores, en específico que llevaron a los alumnos a escoger la licenciatura en Creación y Desarrollo de Empresas, los resultados nos pueden ser útiles para saber si al momento de tomar la decisión los argumentos están acordes a los que se utilizaron para la creación del programa, o su motivación es totalmente distinta a lo planteado.

Siendo más concreto es importante saber qué originó el deseo de los alumnos de contribuir al reconocimiento de la importancia del proceso de emprendimiento, enfocado en el estudio de las motivaciones.

Un elemento digno de tomarse en cuenta es el que aporta Maruland, Montoya, \& Vélez (2019) el cual menciona que: "Aunque se han realizado diversas investigaciones tendientes a encontrar las motivaciones de los emprendedores en diferentes países del mundo, los resultados de éstas no son universales, pues la cultura en la cual nos desarrollamos ejerce una gran influencia en todos los aspectos humanos", por lo que nuestra intención es conocer, en específico, cuál es la motivación de los alumnos de la licenciatura en Creación y Desarrollo de Empresas de la Escuela Suprior de Actopan de la Universidad Autónoma del Estado de Hidalgo. Si bien es cierto que, como hace mención Mayer \& Jiménez (2011), la mayoría de los estudios coinciden que el emprendedurismo va de la mano con un medio eficaz para vincular tecnología, capital y know-how con el fin de aprovechar la habilidad empresarial y acelerar el desarrollo de nuevos proyectos de empresas, pero sin la motivación esto no sería posible.

\section{METOdOLOGÍA}

El objetivo del presente trabajo es conocer de los motivadores que tienen los alumnos de la LCyDE para crear una empresa que tanta similitud tienen con otros países y regiones, por lo que se tomó como referencia la investigación de Maruland, Montoya \& Vélez (Op. Cit.) que citó a Kantis \& Mori sobre su investigación desarrollada sobre las motivaciones de los emprendedores en 13 países de América Latina, el este de Asia y el sur de Europa. A partir de dichos resultados, los autores plantean que las diferencias presentadas indicarían la existencia de contextos culturales muy distintos, como se puede apreciar en la cuadro No. 1, se describe el lugar que ocupan en este ranqueo, así como el porcentaje de emprendedores que consideran importante cada dimensión presentada.

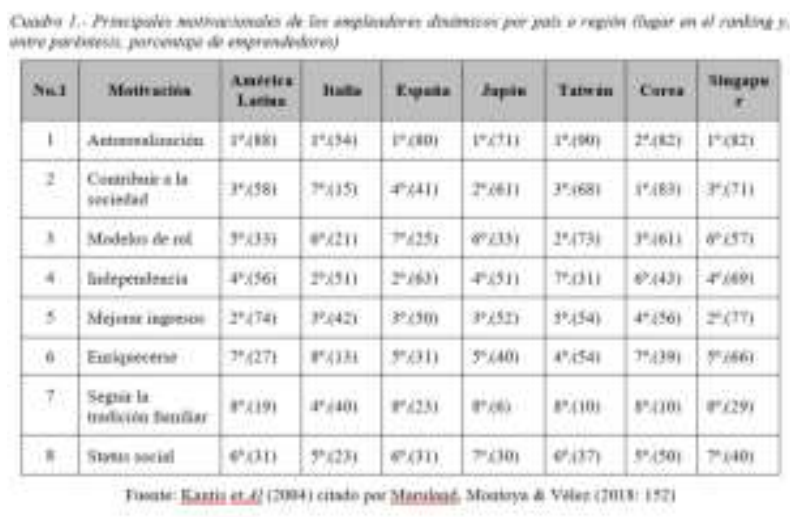

La investigación que se pretende realizar es una investigación no experimental, de diseño transeccional correlacional-causal, con formulación de hipótesis aplicada a una muestra aleatoria simple a los alumnos de la LCyDE que pertenece a la Escuela Superior de Actopan de la UAEH.

Como base hemos tomado la tabla anterior de Marulanda, Montoya \& Vélez (Op. Cit.: 152), que, como ya lo hemos mencionado, se obtuvo de aplicar un instrumento para conocer las principales motivaciones de los emprendedores por país o región, en ocho dimensiones: Autorrealización, Contribuir a la sociedad, Modelos de rol, 
Independencia, Mejorar ingresos, Enriquecerse, Seguir la tradición familiar y Status social.

Por lo que agregamos una nueva "región": los alumnos de la carrera de Creación y Desarrollo de Empresas (CYDE); utilizando, por lo tanto el Método Experimental, que es "aquel que utiliza para comprobar y medir variaciones o efectos que sufre una situación cuando en ella se introduce una nueva causa, dejando las demás causas en igual estado (Eyssautier, 2008)".

Como primer paso se calculó la muestra, la cual la podemos obtener utilizando la fórmula que nos presenta Netquest $\AA$ (Gráfico No. 1):

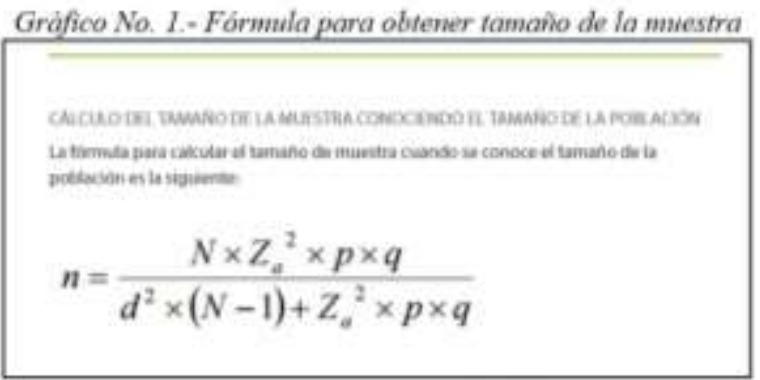

Fuente: Netquest 2018 ).

Otra alternativa es utilizando el software que tiene Mitofsky (2019) para tal fin, con los siguientes datos: la carrera cuenta con 161 estudiantes, utilizamos un margen de error del $5 \%$ y un nivel de confianza del $98 \%$, por lo que se obtuvo un tamaño de muestra de 124 alumnos, el cual va a ser una parte representativa de la población (Nolberto \& Ponce, 2008), en el siguiente gráfico, la número dos, se aprecia la aplicación del software:

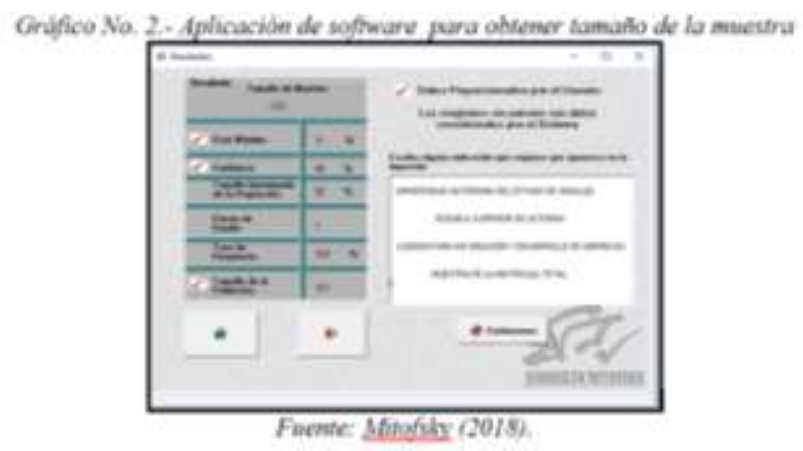

Se tomó de forma aleatoria (Greener, 2008) a los alumnos que contestaron el instrumento, a continuación se presentan algunas imágenes de la aplicación (Gráfico No. 3):

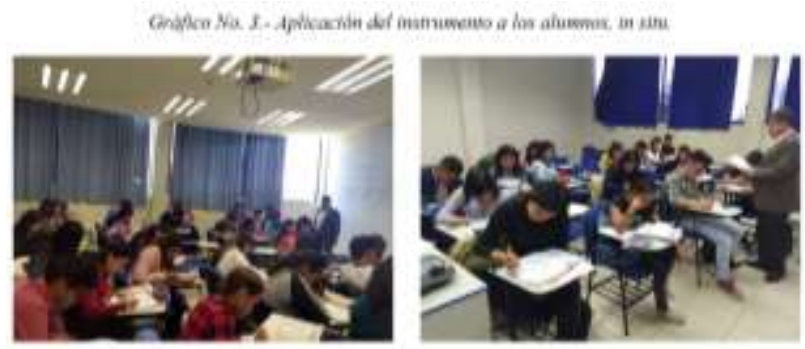

Como siguiente paso, buscamos la asociación entre dos variables (Castilla \& Cravioto, 1999) que vamos a contrastar es decir, la carrera de LDYCE con América Latina, ya que es la región a la que pertenecemos y presumimos que es con la que tenemos mayor afinidad respecto a los motivantes, los porcentajes obtenidos los convertimos a decimal para facilitar el trabajo (Mendoza \& Block, 2010), en la siguiente impresión de pantalla del software Excel® que es el cuadro número dos, se aprecian los datos capturados:

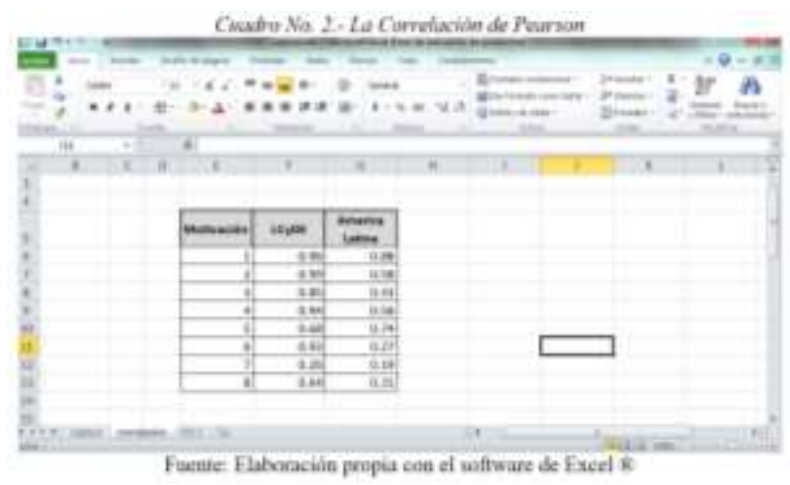

Con estos datos empleando la prueba estadística de ChiCuadrada (X2), la cual sirve para evaluar hipótesis acerca de la relación entre dos variables (Hernández, Fernández, \& Baptista, 2003), ya que el principal propósito de investigación por medio de la estadística inferencial consiste en poner a prueba la hipótesis de investigación por medio de la comprobación de hipótesis estadísticas (Kerlinger \& Lee, 2002) que es la prueba que a continuación se va a desarrollar.

En primer término se obtiene la siguiente hipótesis de investigación:

$\mathrm{H}_{\mathrm{i}}=$ La motivación de los alumnos de la LCyDE para crear una empresa está relacionada con la motivación de los emprendedores de América Latina de crear una empresa

Y como hipótesis nula:

$\mathrm{H}_{0}=$ La motivación de los alumnos de la LCyDE para crear una empresa no tiene relación con la motivación de los emprendedores de América Latina de crear una empresa 


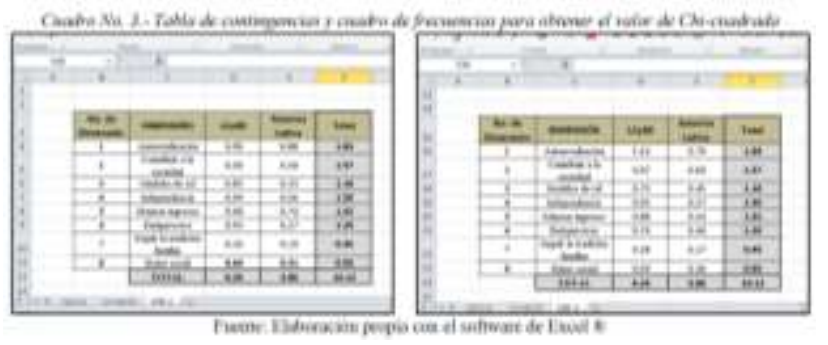

Como ya lo hemos mencionado, para saber si se cumple nuestra hipótesis utilizaremos el test de Chi-cuadrada $\left(\mathrm{X}^{2}\right)$, por lo que elaboramos una tabla de contingencia de dos entradas: la primera respecto a nuestros dos grupos: los alumnos de la LCyDE y la región de América Latina y la segunda las ocho motivaciones de los emprendedores propuestas por Maruland, Montoya \& Vélez (Op. Cit.) que se incluyeron en el instrumento que se aplicó a los alumnos, por lo que en el cuadro número tres se pueden apreciar los dos pasos que se requieren:

La fórmula para obtener el valor de Chi-cuadrada es:

$X^{2}=\sum((0)(E))^{2} / E$

Por lo que utilizando el Excel® obtenemos su valor:



Fuente: Elaboración propia con el software de Excel $\mathbb{R}$

Sustituyendo los valores tenemos: $\mathbf{X}^{2}=\mathbf{0 . 3 8 4 1}$ (valor experimental) y al ser una columna de 8 alternativas y 2 renglones se obtienen los Grados de Libertad (Anthony, 2011) de la fórmula: $G L=(r-2)(c-8)$, donde sustituyendo los valores se obtiene: $G L=(2-1)(8-1)=(1)(7)=7$. Buscamos en la tabla y con un nivel de confianza de 0.05 y 7 grados de libertad:

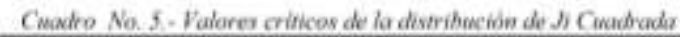

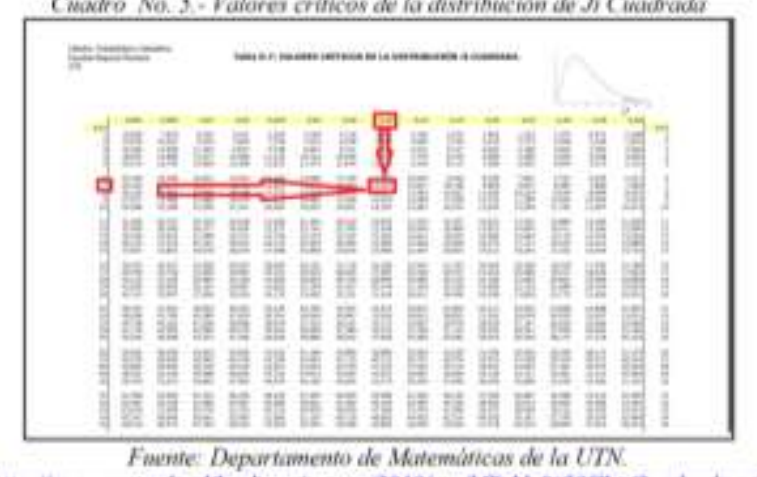

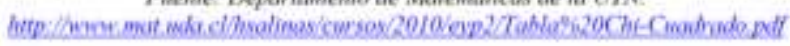

El valor obtenido es de 14.067, que es mayor al valor experimental de 0.3841 . Al ser $X^{2}$ igual o superior a la tabla, decimos que las variables no están relacionadas (Op. Cit. Hernández: 562). Lo que quiere decir que no se cumple la hipótesis de investigación.

Por lo tanto la que se cumple es la hipótesis nula: $\mathrm{H}_{0}=\mathrm{La}$ motivación de los alumnos de la LCyDE para crear una empresa no tiene relación con la motivación de los emprendedores de América Latina de crear una empresa. Entre los valores de ambos grupos, América Latina y los alumnos de la licenciatura en Creación y Desarrollo de Empresas, las dos dimensiones donde existe mayor discrepancia, es decir, mayor diferencial son: en que las empresas deben de contribuir a la sociedad y número dos que éstas son una forma de generar enriquecimiento a los dueños; en ambos casos los valores más altos lo tienen los alumnos de la licenciatura en Creación y Desarrollo de Empresas.

Ambas respuestas representan un aspecto positivo en los alumnos, en el primer caso: que las empresas deben de contribuir a la sociedad resulta muy importante, ya que la derrama económica no es sólo para los empleadores, sino que se pueden obtener beneficios tanto económicos como de bienestar para la comunidad al ser una Empresa Socialmente Responsable, donde los empresarios estén conscientes de que sus acciones y operaciones tienen un profundo impacto en sus trabajadores y en la sociedad que les rodea, ya que hay que hacer algo para que ésta influencia sea positiva para construir una sociedad mejor y más sustentable (Barroso Tanoira, 2008).

Por otro lado ver a la empresa como una fuente de riqueza, habla de que no están interesados en crear una empresa de subsistencia, las cuales crean personas que decidieron iniciar una empresa como, quizá, la última opción para ganarse la vida (Torreblanca, 2016), las que desafortunadamente en nuestro país son el $60 \%$ (Zevallos-Vallejos, 2001).

Al no cumplirse la hipótesis planteada, como siguiente paso buscaremos con qué país o región se tiene una mayor afinidad, por lo que se va a medir con los siete países y regiones para ver con cual es más alta la 
correlación, obteniéndose de las siete regiones la Correlación de Pearson. En la siguiente impresión de pantalla de Excel®, que es el cuadro número seis, se presentan los resultados:

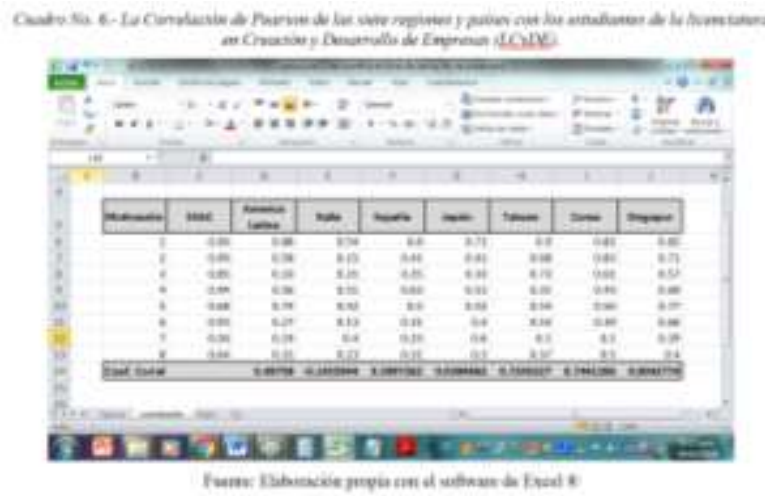

Como se puede apreciar, la correlación más alta que tienen los alumnos de la licenciatura de Creación y Desarrollo de empresas es con los emprendedores de Singapur con una correlación de 0.8042774 la cual es considerada una correlación fuerte (Martínez, Ortíz, Rios, \& Acosta, 2010).

El hecho de que exista una mayor correlación con Singapur nos hace ver algunas de las características de este país, ya que el dinamismo económico de Singapur compensa la falta de recursos naturales. En pocos años, esta nación de sólo 683 km2, con una baja población y con un alto nivel de pobreza, se convirtió en una de las economías más pujantes del Sudeste Asiático adquiriendo el status de New Industrialised Country- NIC (Ramos, 2006). Es uno de los países con más alto acceso a las tecnologías digitales, ya que el $88 \%$ de los hogares tienen internet (ECDL Foundation, 2018). Sobre este mismo tema Marcus Danus, un reconocido emprendedor e inversionistas mexicano, CEO y fundador de Startup México, en una entrevista realizada por García-Fuentes (2018, pág. 24) asegura que "los países que han sido exitosos: Corea del Sur, Malasia, Singapur, Israel o Irlanda, lo han hecho a través de emprendimiento y la innovación, que es una receta probada y funciona perfecto...". También Singapur es la tercer economía en países en desarrollo exportadora (United Nations, 2018). Por ultimo hay que tomar en cuenta que Singapur forma parte de los "Cuatro Tigres del Sudeste Asiático (Taiwán, Corea del Sur, Singapur y Hong Kong)", quienes en las últimas décadas sorprendieron al mundo, por su desarrollo económico, así como por sus avances tecnológicos, dejaron a un lado su realidad de economías agrícolas, pasando a ser, piezas clave en el escenario económico internacional, como países industrializados (Gonzalez-Chávez, 2010).

\section{CONCLUSIONES}

Durante la aplicación del instrumento, además de lo planteado, en un ítem se deseaba saber si en un momento de su vida los alumnos estaban dispuestos a abrir una empresa, donde el $100 \%$ dijo que sí, algo que resulta positivo, ya que la carrera tiene dos vertientes: la creación y el desarrollo; por lo que algunos podrían optar sólo por el desarrollo de las ya existentes. Otro hallazgo es que el $26 \%$ tiene la cultura empresarial por medio de un negocio familiar que desean seguir desarrollando por su cuenta. Después de analizar el cambio radical que ha tenido Singapur en las últimas décadas y esa correlación tan fuerte que tiene con los alumnos de la licenciatura en Creación y Desarrollo de Empresas, nos genera una esperanza de que cuando egresen los estudiantes van a crear empresas con un cambio de mentalidad, mucho más positivo y esto pueda repercutir en un mayor desarrollo en nuestra región. Por lo que consideramos muy importante y positivo que las motivaciones de los alumnos de la LCyDE para crear una empresa sean similares a las de los emprendedores de este país asiático.

\section{RECOMENDACIONES}

Zunzunegui (2018) comenta que Singapur es más pequeño que la ciudad de México y más rico que todo México, no tiene petróleo, de hecho no tiene ningún recurso natural, todo, absolutamente todo, lo importa, incluyendo el agua potable. Han apostado al comercio y la tecnología, lo cual les da suficiente dinero para hacerse de todos los recursos que no tienen. También es un país conquistado en este caso por Inglaterra, y se independizó apenas en 1963 para pasar a ser parte de Malasia de quien se independizó en 1965. Su único gran recurso renovable, vital para el progreso: es la educación. Por lo que es la mejor inversión que podemos hacer en nuestros jóvenes, y fortalecer en lo que somos competitivos, evitando destinar recursos a productos y servicios que sólo se mantienen por tradición pero jamás nos otorgarán las utilidades necesarias.

\section{REFERENCIAS BIBLIOGRÁFICAS}

Alonso, D., González, N., \& Nieto, M. (julio-septiembre de 2015). La innovación social como motor de creación de empresas. Universia Business Review(47), 48-63.

Anthony, D. (2011). Statistics for Health, Life and Social Sciences. United States of America: Ventus Publishing ApS.

Barroso Tanoira, F. (2008). La responsabilidad social empresarial. Revista Contaduría y Administración(226), 73.

Bonilla, R. (enero-marzo de 2014). Innovación empresarial. Arte y ciencia en la creación de empresas. Revista Tecnura, 18(39), 198.

Castilla, L., \& Cravioto, J. (1999). Estadística Simplificada. Distrito Federal, México: Trillas.

CONACyT. (6 de octubre de 2019). Qué es el RENIECyT? Obtenido de https://www.conacyt.gob.mx/index.php/comunicacion/comunicadosprensa/10-contenido-estatico/51-reniecyt 
Díaz, J., Urbano, D., \& Hernández, R. (2005). Teoría económica institucional y creación de empresas. Investigaciones Europeas de Dirección y Economía de la Empresa, 11(3), 209-230.

ECDL Foundation. (2018). Perception \& Reality: Measuring digital skill gaps in Europe, India and Singapore. Dublin, Republic of Ireland: European Computer Driving Licence Foundation.

Eyssautier, M. (2008). Metodología de la Investigación: Desarrollo de la inteligencia. México, D. F. México: CENGAGE Learning.

García-Fuentes, M. (mayo de 2018). Los changarritos deben trascender. Entrepreneur, 26(5), 24-25.

Garcia, S. (2011). El impacto de las incubadoras de empresas en la creación y desarrollo de las MiPYMES en México. Tesis para obtener en título de Licenciado en Economía. Distrito Federal., México, México: Universidad Nacional Autónoma de México.

Gasca, B. (abril de 2018). Adopta una Pyme, de Walmart, hace crecer tu empresa. Revista Entrepreneur, 26(4), 10.

Gonzalez-Chávez, J. (2010). Los cuatro tigres asiáticos. Ciudad de México: Dirección de Servicios de Investigación y Análisis. Subdirección de Política Exterior. Cámara de Diputados. Recuperado el 20 de enero de 2019, de http://www.diputados.gob.mx/sedia/sia/spe/SPE-CI-A-11-10.pdf

Greener, S. (2008). Business Research Methods. United States of America: bookboon.com.

Hernández, A. (2010). La creación y consolidación de empresas sociales a través del instrumento de apoyo al desarrollo empresarial. Tesis de licenciatura en Economía, 15-18. Ciudad de México, México: Universidad Nacional Autónoma de México (UNAM). FES Aragón.

Hernández, R., Fernández, C., \& Baptista, P. (2003). Metodología de la Investigación. Distrito Federal, México: Mc Graw Hill.

Herrera, J., Salas, L., Domínguez, G., \& Torres, K. (julio-diciembre de 2015). Parques científicos-tecnológicos y modelo triple-hélice. Situación del Caribe colombiano. Revista Entramado, II(2), 120.

Kerlinger, F., \& Lee, H. (2002). Investigación del comportamiento. Distrito Federal, México: Mc Graw Hill.

Márquez, L. (junio de 2018). Bitácora. Entrepreneur, 26(6), 19.

Martínez, M., Ortíz, R., Rios, H., \& Acosta, R. (2010). Análisis de correlaciones en poblaciones cubanas de maíz. Cultivos Tropicales, 31(2), 5. Maruland, F., Montoya, I., \& Vélez, J. (enero-junio de 2019). El individuo y sus motivaciones en el proceso emprendedor. Revista Universidad y Empresa, 36(21), 151 y 152.

Mayer, E., \& Jiménez, K. (octubre-diciembre de 2011). Las incubadoras de negocios en México: un análisis descriptivo. Revista CienciaUAT, 6(2), 20911.

Mejía, J. (noviembre de 2012). Modelo de creación de empresas de tecnología, basadas en el perfil del científico-emprendedor. Tesis para obtener el grado de Maestría en Ciencias de Administración de Negocios. Distrito Federal, México: Escuela Superior de Comercio y Administración del Instituto Politécnico Nacional.

Mendoza, T., \& Block, D. (2010). El porcentaje: lugar de encuentro de las razones, fracciones y decimales en las matemáticas. Revista Latinoamericana de investigación en Matemática Educativa. RELIME, 13(4-1), 182.

Nolberto, V., \& Ponce, M. (2008). Estadística Inferencial Aplicada. Lima, Perú: Unidad de Posgrado de la Facultad de Educación de la Universidad Nacional Mayor de San Marcos.

Quintero, A. (2003). El aprendizaje en la empresa: la nueva ventaja competitiva. Revista Educación y Educadores(6), 133.

Ramos, J. (06 de mayo de 2006). Economía y Comercio: Singapur. (U. d. Barcelona, Productor) Obtenido de Master en Comercio y Finanzas Internacionales: https://www.comercioexterior.ub.edu/correccion/0506/singapur2006/7.2.econpolt.ecocomer.htm

Reynoso, C. (junio de 2014). Las transformaciones del concepto de empresa. Latinoamericana de Derecho Social(18), 133-158.

Rodeiro, D., Fernández, S., Otero, L., \& Rodríguez, A. (2008). La creación de empresas en el ámbito universitario: una aplicación de la teoría a los recursos. Cuadernos de Gestión, 8(2), 11-28.
Rodríguez, M., Acuña, J., Rojas, L., \& Lobato, L. (2015). Vinculación Universidad-Empresa-Estado, en Nicaragua. Fundación Miguel Unamuno y Jugo. Revista Científica de Ciencias Humanas, 11(31), 17-18.

Santamaría, C., \& Brunet, I. (julio-diciembre de 2014). Creación de empresas y spin-off universitarias en México. Revista Iberoamericana para la Investigación y el Desarrollo Educativo, 5(9), 8 y 11.

Torreblanca, E. (14 de noviembre de 2016). Microempresas, asunto de seguridad nacional. Recuperado el 11 de enero de 2019, de https://www.elfinanciero.com.mx/opinion/eduardo-torreblancajacques/micro-empresas-asunto-de-seguridad-nacional

UAEH. (2005). Guía para el Rediseño y Presentación de Programas de Licenciatura. Universidad Autónoma del Estado de Hidalgo, 235-238. Pachuca, Hidalgo, México.

UAEH, ,. (2014). Diseño Curricular de la Licenciatura en Creación y Desarrollo de Empresas. Escuela Superior de Actopan de la Universidad Autónoma del Estado de Hidalgo. Pachuca, Hidalgo, México.

United Nations. (2018). World Investment Report 2018. Investment and New Industrial Policies. New York: UNCTAD.

Uribe, E. (febrero de 2018). ¿Tienes el ADN emprendedor? Revista Entrepreneur, 26(2), 34.

Zevallos-Vallejos, E. (diciembre de 2001). Empresa media, una nueva clasificación. Comercio Exterior, 1101. Obtenido de http://revistas.bancomext.gob.mx/rce/magazines/29/8/RCE.pdf

Zunzunegui, J. (2018). Los mitos que nos dieron patria. (Undécima ed.). Ciudad de México, México: Penguin Random House Grupo Editorial, S. A. de C. V.

Zúñiga, A. (2013). Incubadoras de Negocios: en la creación de empresas competitivas. Revista Entretextos, 46. 